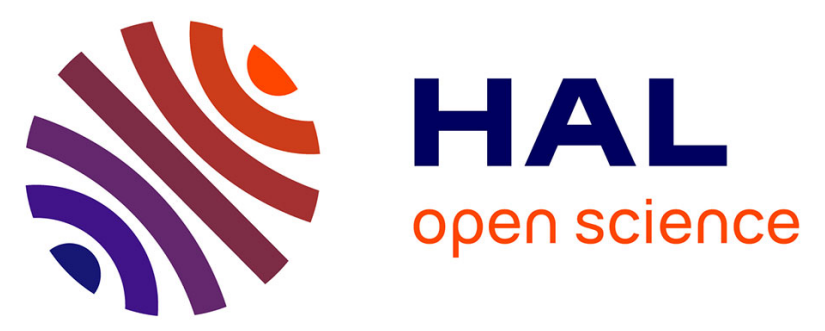

\title{
A Kinetic Monte Carlo Algorithm to Model the Annealing Process and Compute the Dark Current Nonuniformity
}

Kevin Lemiere, Christophe Inguimbert, Thierry Nuns

\section{To cite this version:}

Kevin Lemiere, Christophe Inguimbert, Thierry Nuns. A Kinetic Monte Carlo Algorithm to Model the Annealing Process and Compute the Dark Current Nonuniformity. IEEE Transactions on Nuclear Science, 2021, 68 (8), pp.1701-1711. 10.1109/TNS.2021.3074369 . hal-03348968

\section{HAL Id: hal-03348968 \\ https://hal.science/hal-03348968}

Submitted on 20 Sep 2021

HAL is a multi-disciplinary open access archive for the deposit and dissemination of scientific research documents, whether they are published or not. The documents may come from teaching and research institutions in France or abroad, or from public or private research centers.
L'archive ouverte pluridisciplinaire HAL, est destinée au dépôt et à la diffusion de documents scientifiques de niveau recherche, publiés ou non, émanant des établissements d'enseignement et de recherche français ou étrangers, des laboratoires publics ou privés. 


\title{
A Kinetic Monte Carlo Algorithm to Model the Annealing Process and Compute the Dark Current Nonuniformity
}

\author{
K. Lemière, C. Inguimbert, T. Nuns
}

\begin{abstract}
A simulation chain, composed of GEANT4 and a Kinetic Monte Carlo algorithm, dedicated to study the annealing process of defects produced after energetic particle irradiation is presented. Results will be used to compute Dark Current Nonuniformity.
\end{abstract}

\section{INTRODUCTION}

$\mathrm{D}$ uring energetic particle irradiation, like in space or in particle accelerators, electronic devices could suffer from different types of damage. Displacement of atoms causes some specific degradations in semiconductor materials [1]. Displacement Damage Dose (DDD) consists in the mean energy deposited by nonionizing process. It causes displacement of atoms surrounding the incident particle track. Depending on the energy involved in the nuclear reaction, isolated defects or cluster of defects are produced [2]. When an incident particle displaces a lattice atom, the latter is called a Primary Knock-on Atom (PKA). If the PKA transmits enough energy to displace a neighboring atom, it is called a Secondary Knock-on Atom (SKA). Damage cascades are created through these processes. After a short time, displaced atoms could get back to a lattice position, or be definitively kicked out of their lattice position: this is called a Frenkel pair, a pair of an interstitial atom and an associated vacancy [3]. Following this initial phase, some Frenkel pairs recombine almost instantaneously after the thermal energy has dissipated (thermal spike). Then, primary defects start moving out through the semiconductor lattice thanks to their thermal energy. During this diffusion process, a defect can encounter another defect. Depending on the nature of colliding defects, they could either recombine, or associate in a more complex one. Finally, the small fraction of defects which survived could lead to the introduction of electrically active deep or shallow trap levels in the semiconductor band gap [4]. The deep trap levels are responsible of the increasing number of charge carriers produced in the depleted region observed after an irradiation in detectors. It results in an increase of the intrinsic parasitic current, the so-called dark current.

In addition, energetic particle irradiation is uniform but the associated damage production is not uniform. In imagers, this leads to statistical variation of degradation undergone by each pixel: this is called the Dark Current Nonuniformity (DCNU) [5]. In this paper, a simulation chain composed of different numerical tools to compute the DCNU created by proton irradiation is presented. This toolkit relies on the assumption of proportionality between the increase in dark current and the DDD by means of a universal damage factor [6]. In order to avoid the use of this empirical parameter

K. Lemière, T. Nuns and C. Inguimbert, are with ONERA-DPHY, 2 avenue E. Belin, 31055 Toulouse, France

(Phone: 33 (0) 562252568, email: kevin.lemiere@trad.fr). which not takes into account an accurate annealing state, we have developed a numerical chain capable to estimate annealing rates and nature of surviving defects. Knowing their thermal emission rate we are able to estimate the increase in the DCNU induced by an irradiation. Generation of damage cascade tracks, simulation of annealing process and DCNU computation are treated separately in order to avoid too complex and time-consuming simulations. Each simulation step is run from input data calculated from the previous one. Firstly, the GEANT4 toolkit [6] is used, in order to have a realistic spatial distribution of Frenkel pairs in the volume. Each track is stored in a database. Then, these defect distributions are used as input for a Kinetic Monte Carlo (KMC) algorithm [8]. In this paper, the KMC algorithm is dedicated to the estimation of annealing process that occurred in a defect distribution. In other words, the KMC algorithm is used to estimate which stable defect population could be expected at a given time after irradiation.

A statistical study is made on the annealing factor and the final population of complex defects by executing numerous KMC simulations. Finally, introduction of KMC simulation results as input of our Monte Carlo method, dedicated to the prediction of dark current distribution through a pixel array, is presented in this paper.

\section{GEANT4 CASCADE DAmage Simulations to CREATE KMC INPUT DATA}

\section{A. GEANT4 simulation framework}

When an energetic particle creates a damage cascade in the matter, the shape of the cascade and the number of defects produced are not the same for each incident particle. In the aim of having the most realistic annealing simulation, GEANT4 code has been used to simulate the damage cascade caused by a PKA according to the Binary Collision Approximation (BCA) in a simulation box exclusively composed of silicon. Below approximately $10 \mathrm{keV}$ incident heavy ions, the BCA is close to limit of validation and alternative simulations such as full Molecular Dynamic must be preferred.

The GEANT4 version used in this work is 9.6 patch 03 , with the threshold displacement damage energy set to $21 \mathrm{eV}$. The G4ScreenedNuclear module has been used to model the nuclear interactions between the incident energetic particle and the silicon atoms. The simulation volume is a box with sides of some microns. The punctual particle source is placed inside the simulation volume, a few dozen of nanometers under the surface of the box, in order to keep tracking atoms that have been kicked out in the reverse direction of the incident energetic particle direction of propagation. Position of each vacancy and interstitial created during the simulation is stored. The vacancy location 
corresponds to the initial position of a displaced atom, and the associated interstitial location corresponds to the position where the displaced atom has zero kinetic energy. 1,000 simulations are executed to have a sufficient database of damage cascades. In other words, 1000 PKA with the same energy and same incident direction (normal direction to the surface of the box) are considered for GEANT4 simulations.

\section{B. Choice of the nature of simulated incident particle in GEANT4}

In the frame of this work, we focused exclusively on dark current degradation caused by energetic proton irradiation. Even if it may seem intuitive at first to perform a KMC simulation on all the defects produced along the trajectory of a proton, this strategy can be highly time consuming. Indeed, a proton will generate a set of PKA on dimensions that imposes prohibitive computation times. The damage cascade produced by a single PKA can contain thousands of vacancy-interstitial pairs, which can already be long in computation time. Simulating the trajectory of a proton would lead to days or even weeks of simulation in the worst case. In order to avoid these constraints, we chose a different approach.

If one assumes that the damage cascades of each PKA are independent, and this is almost the case, since the PKA are produced at sufficiently large distances compared to the size of a sub-cascade, a more time-efficient calculation strategy consists in simulating a damage cascade from a PKA and not from the incident proton. Rather than simulating the entire trajectory caused by an energetic particle, only part of the cascades is modeled by choosing a recoil nucleus as the precursor of the cascade. This approach is justified by the fact that one can consider that a cascade is a set of subcascades created by the different PKA. This is a classic strategy also adopted by Raine et al. [9] and Jay et al. [10], [11].

\section{Choice of the PKA energy to simulate in GEANT4}

In their work, Wood et al. [12] estimate that $6 \mathrm{MeV}$ to $10 \mathrm{MeV}$ protons induce $1 \mathrm{keV}$ to $2 \mathrm{keV}$ PKAs. The authors estimate also that protons of more than $20 \mathrm{MeV}$ generate PKAs in the range of $12 \mathrm{keV}$ to $20 \mathrm{keV}$. Srour et Palko [1] consider that $100 \mathrm{MeV}$ protons create PKAs in the order of $10 \mathrm{keV}$. More precisely, Inguimbert et al. [13] calculate the atoms recoil energy after proton irradiation and confirm most of these PKA energy ranges. The authors find that protons from $1 \mathrm{MeV}$ to $100 \mathrm{MeV}$ generate PKAs in the range of $1 \mathrm{keV}$ to approximately $100 \mathrm{keV}$. The choice of the PKA energy are essentially based on the PKA energy distribution provided by Inguimbert et al. [13]. GEANT4 proton simulations have been performed for the presented work and are in agreement with this PKA energy range.

Finding a typical energy for a PKA produced by a $\mathrm{p}+\mathrm{Si}$ interactions and reducing the population of various PKAs to a single typical particle is not possible and can only be an approximation to the problem. We were forced to do that for obvious CPU time reasons. PKAs can be produced by both Coulombian and nuclear reactions. If we focus on high $\mathrm{Z}$ recoil nuclei produced by nuclear reactions the distribution of energy are comparable to Gaussian for which an average value of several $\mathrm{MeV}$ can be defined easily [14]. But most of recoil nuclei are produced by Coulombian interactions that are far more numerous. Such kind of interactions present strong decreasing distributions having a very low average energy $(\sim \mathrm{keV}) .100 \mathrm{keV}$ is a kind of trade off to get a sufficient high energy which could be representative of nuclear recoil $(\sim \mathrm{MeV})$, a not to high energy to get something representative of Coulombian interactions and which is compatible with KMC simulations durations. This assumption is supported by the fact that for higher PKA energies the damage cascade presents independent subcascades of lower energy recoils.

As a consequence, the PKA energy that will be treated in the Kinetic Monte Carlo program is chosen as a function of the proton energy. If the proton energy is below $10 \mathrm{MeV}$, the KMC algorithm will receive $1 \mathrm{keV}$ PKA damage cascades as input. If the proton energy is greater than 10 $\mathrm{MeV}$, the PKA energy considered is $10 \mathrm{keV}$. For more energetic protons (above $100 \mathrm{MeV}$ ), the PKA energy is fixed to $100 \mathrm{keV}$.

In order to model the reorganization of the matter after a proton irradiation, i.e the annealing of the PKA damage cascade, the Kinetic Monte Carlo (KMC) algorithm has been selected. In the following, the algorithm used in this work is presented, as well as the parameters used to model the diffusion of primary and complex defects in silicon.

\section{Kinetic MONTE CARlo SimUlations}

Recently, Raine et al. and Jay et al. [9]-[11] proposed a complex chain of simulation to model the creation of stable electrically active defects in silicon, coupling molecular dynamics (MD) simulations, a k-ART technique and $a b$ initio calculations.

The k-ART technique relies on a complex KMC algorithm, which construct a library of possible event at each step. To be more precise, the k-ART technique refines the energy barriers taken from the library as a function of the surrounding defects. This way, the algorithm is more realistic, but need a heavy computational cost.

In this paper, the authors investigated the influence of different defect switching states, i.e. defects that have different levels in the band gap and switch between those, on electrical measurements made after irradiation, and proposed hypothesis to explain the creation of the Random Telegraph Signal (RTS) pixels. Their work proposes accurate simulations at each timescale of the simulation, corresponding to different phases of the physical process. The initial damage cascade is simulated with GEANT4. Then the thermal spike is simulated thanks to MD simulations in order to get accurate defects characteristics just a few picoseconds following the initial damage cascade at atomic level. From these characteristics, a KMC based algorithm is used to model the annealing processes for longer time durations.

In our work, we did not concentrate on the prediction of RTS levels, but on the realistic estimation of defects mainly responsible of the dark current level enhancement observed after irradiation, followed by a long-term annealing. Our goal is to include a relaxation step to our DCNU modelling and in this way removing the use of the empirical parameters. But for that, we needed to compute a large number of damage cascade for various PKA energies. In order to achieve this goal, as following the kinetic of the displacement damage cascades is CPU consuming, we have chosen to develop a KMC algorithm, less accurate method 
than some industrial code [15] or advanced scientific code [9]-[11] but faster.

We focused on the KMC simulations in order to access to the final population of damages few days up to 1 month after irradiation. The main goal of this approach is to provide realistic input defect data to our Monte Carlo toolkit dedicated to the computation of the Dark Current Nonuniformity. MD simulations are not used in this work but the migration energy barriers computed in [10], [16], that determines the kinetic properties of each defect (see Table 1 in section III.C), are used in the KMC algorithm developed in this work.

\section{A. The Kinetic Monte Carlo Algorithm}

The KMC algorithm is used to create a database of annealing factor according to PKA energy (i.e a distribution of annealing factors according to the KMC results), and also to estimate the final population of complex defects. This information will then be used as input for the Monte Carlo based simulation toolkit dedicated to the computation of the Dark Current Nonuniformity on a whole pixel array (named $D A A N$ [17]), and then compared with experimental data. The annealing factor defines indirectly the quantity of defects (single or complex) that vanishes (anneals) at a given time after irradiation. The annealing factor $A F$ is simply expressed as:

$$
A F=\frac{N b . o f \text { defects at final state }}{N b . \text { of defects at initial state }}
$$

The final population of complex defects, or more simply trap population, defines the distribution of traps in the simulation, i.e. in what proportions are present each species of defect in the simulation.

The developed off-lattice KMC method is based on the model described by Nordlund [18]. The defect's kinetic is directly linked to three physical parameters: the migration energy $E_{m}$, the intrinsic jump frequency $w_{0}$ and the lattice temperature $T$. The two former parameters are defined by the nature of the defects. Migration energy is equivalent to the potential barrier that a defect must overcome to make a jump in the lattice, i.e move to another location in the lattice. For a given defect $i$, the intrinsic jump frequency $w_{0, i}$ designates the number of attempts made by the defect to overcome the potential barrier by unit of time. From these parameters, the term $W_{i}$ is deduced, which corresponds to the number of jumps that a defect can make during a unit of time, and computed as follows:

$$
W_{i}=w_{0, i} \exp \left(\frac{-E_{m, i}}{k_{B} T}\right)
$$

The jump process is a thermally activated mechanism, that is why the expression of $W_{i}$ is similar to an Arrhenius law. It could be associated to a diffusivity or a defect mobility.

The key steps of the Kinetic Monte Carlo algorithm, inspired from [18], are detailed in the following:

$\left.1^{\circ}\right)$ Firstly, all primary defects are placed in the simulation box according to a GEANT4 PKA damage cascade simulation. The time simulated in the KMC algorithm is initialized $(t=0)$. A defect list is established, containing there coordinates in the simulation volume, their name and their migration properties (migration energy, intrinsic jump frequency and frequency jump). $2^{\circ}$ ) The algorithm checks if some Frenkel pairs are close enough to recombine directly. In practice, this step is equivalent to test if a vacancy and an interstitial are within the interaction radius of vacancy-interstitial pair defined by the user to initiate a recombination process. In our simulation, the recombination radius is fixed to $4 \AA$ for all defects according. This value is a typical parameter set between the lattice parameter and the distance between the closest silicon atoms in an elementary lattice, according to [10], [18]. It is a first order process, in order to simplify the modeling of the physical mechanism that follows the thermal spike after the crossing of energetic particle. Indeed, a few nanoseconds after that particle deposited its energy, some Frenkel pairs are so closed that they recombine a few moment later, after the thermal energy is dissipated [19]. At the end of this step, the defect list is updated.

$3^{\circ}$ ) The cumulative function $R_{j}$ is computed:

$$
R_{j}=\sum_{i=1}^{j} W_{i}
$$

The sum is made from the first defect $(i=1)$ to the $j$-th defect. The last term is obtained when $j=N$, with $N$ the total number of defects in the system at the beginning of the step. The term $R_{j}$ equals to the sum of each $W_{i}$ of the $j$-th defects of the system at the beginning of the step. $R_{N}$ is noted $R$ for writing simplification.

$\left.4^{\circ}\right)$ A random number $u \in[0,1]$ is drawn uniformly. Then the number $u \times R$ is calculated. Finding the index $i$ where $R_{i} \leq u R<R_{i+1}$ leads to find the defect which will move during the KMC step.

$5^{\circ}$ ) After selecting the defect that will move during the current step, the migration process can start. The defect jumps in a three-dimensional random direction with a radius defined by the user as a KMC input, noted $r_{\text {jump }}$. The direction is defined by two random angles, $\theta$ and $\varphi$, that define a spherical angle. One must precise that our KMC algorithm is an "off-lattice" algorithm, it means that the crystal lattice is not taken into account. As a consequence, no jump direction is preferred.

$\left.6^{\circ}\right)$ At the end of the defect jump, the algorithm tests if there is one or more defects in a specific interaction radius (also entered as KMC input) that could interact with it, noted $r_{i n t}$. If there is more than one candidate in the interaction radius, we suppose in this work that the closest candidate will interact with the defect. Depending on the nature of the candidate, a recombination or association process starts. For example, if a vacancy and an interstitial are within the interaction radius defined by the user, they could recombine and they are both deleted from the system. In the opposite case, if a vacancy is in the interaction radius of another vacancy, they could associate with each other to form a well-known defect: the di-vacancy.

$7^{\circ}$ ) Finally, the list of defects and their properties is updated, and the migration time step is determined randomly through the KMC characteristic relationship: 


$$
\Delta t=-\frac{\log u^{\prime}}{R}
$$

where $u^{\prime}$ a random number $u^{\prime} \in[0,1]$ drawn uniformly. At the end of the step, the simulated time is updated, the algorithm is repeated from the step $3^{\circ}$ ) and a new step begins until the stopping criterion is reached. The stopping criterion can be for example a maximum number of iterations or a maximum simulated time in KMC. It is important to underline the fact that only one defect moves at each iteration of the algorithm.

Use of KMC algorithm implies that a defect selected randomly according to its jump probability moves in a random direction during a random migration time, and could interact with a surrounding defect. The most powerful advantage of using KMC algorithm is its adaptability to a large range of timescale. Indeed, as the simulation runs, the time step $\Delta t$ evolves as function of the number of defects in the system and their jump frequency. In addition, a great advantage of KMC algorithm is to be able to take into account these different kinetics to make evolve the system. Indeed, at each step of KMC simulation, a defect is selected according to its jump frequency. Hence, the algorithm ensures that the most mobile defects are selected more often than the least mobile defects.

In order to avoid too much time consuming simulations, the size of the simulation box is "adaptative". It means that the size of the box is proportional to the size of the damage cascade. We choose to manage the size of the simulation box in this way because the simulation cost can be very expensive if the lonely vacancies and interstitials diffuse in a too large box. Hence, for each damage cascade the largest $\mathrm{X}$ coordinate is multiplied by 10 , the largest $\mathrm{Y}$ coordinate by 10 , and the largest $Z$ dimension "only" by 5 , because the PKA is launched along the depth- $Z$ axis and the damage cascade has more chance to extend along the projectile incident direction. Following this idea, the simulation box is similar to a cuboid of a dozen to a hundred of nanometers for $1 \mathrm{keV}$ or $10 \mathrm{keV}$ PKA.

\section{B. Defect interaction management}

To run a classical KMC algorithm, the user needs to define some rules and exceptions to deal with the different interactions (recombination or association) that can occurs during the simulation. In this work, an "authorized" species list is created. This list indicates to the KMC algorithm which defect it is able to create and under which conditions.

To activate an interaction between two species, the distance separating them has to be lower than the interaction radius defined for this specific reaction.

During the KMC, different defects could interact through recombination process or association process. A recombination process implies the annihilation of the two defects. Taking the example of an interstitial $I$ and a vacancy $V$, we obtain:

$$
I+V \rightarrow \varnothing
$$

In this work, the association process occurred when two defects of the same species interact together. Here the classical relationship of di-vacancy creation:

$$
V+V \rightarrow V_{2}
$$

A complex defect can also experience a partial recombination, and this is taken into account in our KMC algorithm. For example, if a tri-vacancy (complex of three vacancies) meets an interstitial, it could also create a divacancy:

$$
V_{3}+I \rightarrow V_{2}
$$

When a defect is created, its coordinates are computed as the center of each Cartesian coordinates.

The maximum complex defect size that can be created is limited to 3 elements, i.e $V_{3}$ and $I_{3}$ are the biggest defect that our KMC can create, unless the contrary is explicitly indicated. The maximum complex defect size is voluntarily limited because we are interested into long term annealing (one month after irradiation) and according to the literature, large complex defects seems to be rare on long time period. Indeed, large size clusters (more than 4 interstitials or vacancies approximately) tends to recrystallize or disaggregate [10], [20], [21]. Moreover, the electrical properties of these kind of defects are unknown. As a consequence, the creation of defects involving more than 3 interstitials or vacancies are not permitted in our simulations.

Finally, the search method has been optimized to reduce the computation cost and gain simulation time. Initially, in this kind of algorithm, searching the best candidate (the closest defect in our case) for an interaction is time consuming, because the algorithm has to go through the whole defect list and compute the distance one by one. This process is really time consuming, and in addition it is repeated a large number of times, so this operation is penalizing for the KMC algorithm. For that reason, an octree search method replaces the classical search method in our Kinetic Monte Carlo algorithm. An octree (contraction of "octary" and "tree") search method consists in divided recursively a volume in 8 sub-volumes according to a criteria defined by the user [22], [23]. It could be for example a number of elements in a volume or a maximum depth of the tree. An octree is a hierarchical data structure optimized for searching operation, where generally some libraries are provided to deal with the octree. As a consequence, only the defects contained in the sub-volumes located in the interaction radius are tested. Because there are much less candidates to test, the search method execution time is divided by approximately a factor 4 .

\section{Inventory of the different species considered in the KMC algorithm}

All species considered in this work are summarized in Table 1 . The choice of the value of the migration parameters for each defect is supported by a bibliographic review.

\begin{tabular}{|c|c|c|c|}
\hline Species & $\boldsymbol{E}_{\boldsymbol{m}}(\boldsymbol{e V})$ & $\boldsymbol{w}_{\mathbf{0}}\left(\boldsymbol{f s}^{\mathbf{1}}\right)$ & $\begin{array}{c}\boldsymbol{W}_{\boldsymbol{i}}\left(\boldsymbol{f \boldsymbol { s } ^ { - 1 }}\right) \\
(300 K)\end{array}$ \\
\hline$I:$ mono-interstitial & 0.9 & 1.71 & $1.30 \times 10^{-15}$ \\
\hline$V:$ mono-vacancy & 0.51 & $1.28 \times 10^{-3}$ & $3.47 \times 10^{-12}$ \\
\hline$I_{2}:$ di-interstitial & 0.28 & $10^{-2}$ & $1.98 \times 10^{-7}$ \\
\hline$V_{2}:$ di-vacancy & 1.0 & $10^{-2}$ & $1.59 \times 10^{-19}$ \\
\hline$I_{3}:$ tri-interstitial & 1.66 & $10^{-2}$ & $1.30 \times 10^{-30}$ \\
\hline$V_{3}:$ tri-vacancy & 1.32 & $10^{-2}$ & $6.68 \times 10^{-25}$ \\
\hline
\end{tabular}

Table 1: Each species has their own migration properties. The jump frequency is indicated to identify the most mobile defects. 
It has to be mentioned that these values correspond to "mean" values of the migration parameters. These values can vary from one work to another, depending on the simulation conditions (the shape of the potential driving the interatomic forces) or the experimental protocol [4], [10], [16], [19], [24]-[36]. Nevertheless, the migration parameters used in this work are similar to those used in [10], [16].

In silicon, interstitial and vacancy do not have the same migration energy and intrinsic frequency factor. From room temperature to approximately $1000 \mathrm{~K}$, vacancy is largely more mobile than interstitial. As a consequence, their diffusion kinetics are not the same. According to the parameters chosen for the KMC algorithm, di-interstitial defect has the largest jump frequency, followed by vacancy and interstitial. This is due to their low migration barrier energy, which facilitates their move into the lattice. The migration radius is typically set to $2.35 \AA$, which correspond to the nearest neighbor in a silicon cell. On short term annealing calculations, the value of the migration radius could have an important effect. However, our KMC algorithm is dedicated to long term annealing, so the choice of the radius as less impact on results if the radius value does not vary on several order of magnitude. We consider that on average, the most probable jumping site location is the closest neighbor site. In silicon, it corresponds to a distance of $2.35 \AA$. The typical interaction radius is set to $4 \AA$, which is considered as sufficiently representative of the diverse interaction process after [18]. The more the jump radius increases, the more the defects move on long distance. Also, as the interaction radius increases, the more easily the defects interact between them, because the required distance to trigger an interaction is higher.

\section{USING KMC SIMULATION RESULTS AS DARK CURRENT COMPUTATION INPUT DATA}

In order to know how many defects on average will survive during the annealing process after irradiation and to have an idea of which species could be expected at the end of the annealing, the KMC algorithm described previously is used. Annealing scenario will not be the same if the experience is repeated many times. It is a stochastic process and as a consequence, a statistical study is needed to extract relevant information about these simulations. As the depleted surface of the pixel is approximately equal to $20 \mu \mathrm{m}^{2}$, and a PKA damage cascade spreads typically on a surface of some hundreds of nanometers $\left(400 \mathrm{~nm}^{2}\right.$ on Fig. 1 ), one can consider that the PKA damage cascade can be treated separately.

\section{A. Statistical study of energetic particle damage annealing by simulation with the KMC algorithm}

From our database of 1000 PKA damage cascade simulations for each energy, 30 damage cascades at chosen randomly for $1 \mathrm{keV}$ and $10 \mathrm{keV}$ PKA, only 5 for $100 \mathrm{keV}$ PKA due to high computation time. At the beginning of each simulation, a damage cascade track is randomly selected and the KMC algorithm is applied. Hence, for a given PKA energy, a distribution of annealing factor is obtained. It will serve for the last simulation step: the computation of the Dark Current Nonuniformity. At the end of this computation step, average and standard deviation of the annealing factor distribution are calculated. In addition to this, the number of each species presents at the end of KMC simulation is stored. Thus the information about which species that are the most representative at the end of the simulated annealing process is available. This information will also be used in the next simulation step.

An example of a simulated damage cascade caused by $10 \mathrm{keV}$ silicon PKA is shown in Fig. 1.

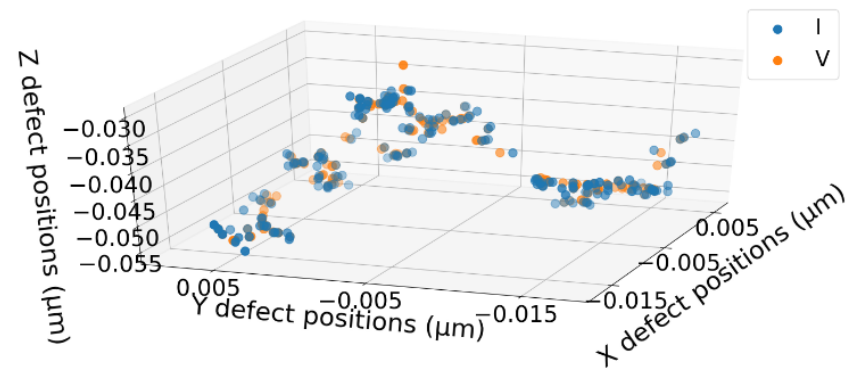

Fig. 1: Initial spatial distribution of Frenkel pairs. This damage cascade results from the simulation of $10 \mathrm{keV}$ silicon PKA in GEANT4. Blue points are interstitial defects, orange one are vacancy defects. The color transparency is linked to the defect position (front side or back side) in the figure. The number of vacancies and interstitials is identical at the initial stage of the simulation. Most of the time, the size of the simulation box is in the order of a dozen of nanometers.

The KMC simulations are made at $294.15 \mathrm{~K}$, because the results will be compare to dark current experimental data obtained at this temperature. Fig. 2 shows result of simulation performed in the case of the initial defect configuration presented in Fig. 1. At the beginning of the simulation, vacancies are so mobile that they start moving in first. It explains the quick decreasing number of vacancies and the increasing production of based vacancy complexes during first microseconds observed in Fig. 3. A tenth microseconds after, interstitials start jumping, and have the possibility to create interstitial based complexes. Triinterstitial and tri-vacancy defects are almost motionless, due to the high migration energy barriers that these defects have to overcome to make a jump. This implies that once one of these defects is created, the probability that it makes a jump is very low. The only way for these defects to evolve is that they encounter an opposite species to do a partial recombination and move again. At the end of the simulation, the final distribution of defects that have been produced during the KMC algorithm is stored.

As the number of each defect species is stored each time an interaction occurred during the KMC execution, the evolution of each species can be observed, as shown in Fig. 3. For example, primary defects, vacancies and interstitials, tend to extinct with time, whereas the number of small complex defects increases with time simulation. Step-like annealing curves have also been observed in [10].

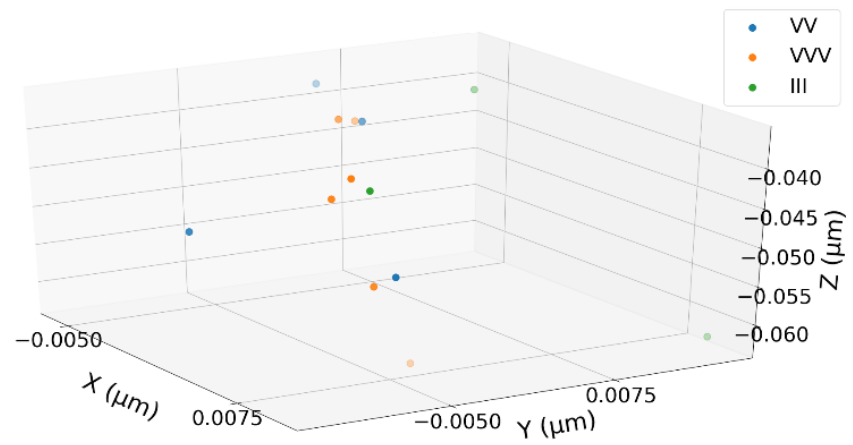

Fig. 2: Final spatial distribution of complex defects. It represents the final state of the Fig. 1 after a KMC simulation of 1 month at 294.15 K. Some divacancies, tri-vacancies and tri-interstitials have been created (blue, orange and green points respectively). 
If a defect moves out the simulation box, it is no longer tracked but it is taken into account in the annealing rate calculation as a recombination, as we consider that it could potentially interact with fictive defects coming from other damage cascades.

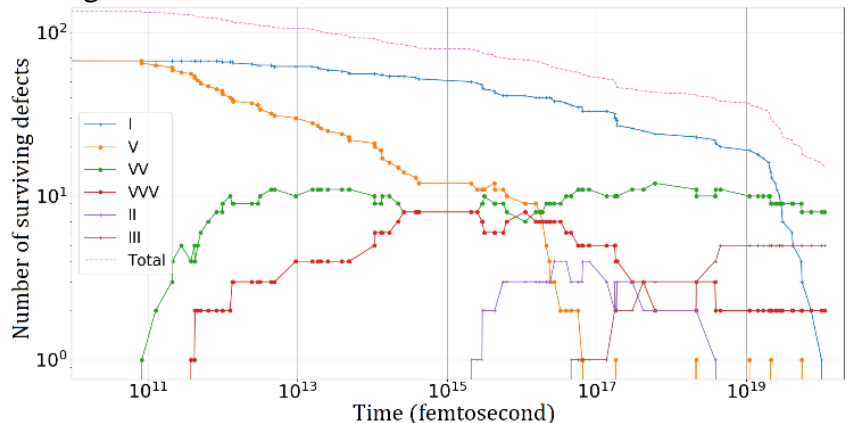

Fig. 3: Evolution of the population of each defect species with time at 294.15 K. As the population of primary defects decreases, the population of more complex defects increases. The transition from the initial state (Fig. 1) to the final state (Fig. 2) is observed from this figure.

The results of the statistical study are summarized in Table 2. The simulations give a mean annealing rate $\left\langle\tau_{\text {anneal. }}\right\rangle$ slightly below $90 \%$ for PKA energy comprised between $10 \mathrm{keV}$ and $100 \mathrm{keV}$ (i.e $10 \%$ of the initial defects survive and create stable defects), a value in agreement with [5]. Concerning the $1 \mathrm{keV}$ PKAs, the mean annealing rate is around $82 \%$, so it is slightly lower compared to $10 \mathrm{keV}$ and $100 \mathrm{keV}$. This discrepancy is due to the higher variation on the mean annealing factor, as we can see from the standard deviation. This variation is caused by the nature of the damage cascade. A $1 \mathrm{keV}$ PKA is likely to cause isolated defects along its track. As a consequence, the primary defects have more chance to get out the simulation box and they do not associate with others defects. For more energetic PKAs, the damage cascades tend to create high defect density pockets, where the defects are closer each other and interact more easily.

\begin{tabular}{|c|c|c|c|}
\hline & $1 \mathrm{keV}$ & $10 \mathrm{keV}$ & $100 \mathrm{keV}$ \\
\hline$\left\langle\tau_{\text {init. rec. }}\right\rangle(\sigma)$ & $58.6 \%(7.7 \%)$ & $57 \%(2.5 \%)$ & $56.6 \%(1 \%)$ \\
\hline$\left\langle\tau_{\text {anneal. }}\right\rangle(\sigma)$ & $81.8 \%(5.5 \%)$ & $87 \%(2 \%)$ & $88.8 \%(1 \%)$ \\
\hline
\end{tabular}

Table 2: Mean initial recombination rate and mean annealing rate for different PKA energies after 1 month KMC simulations at 294.15 K. The standard error is mentioned into brackets. 30 simulations have been done for $1 \mathrm{keV}$ and $10 \mathrm{keV}$, but only 5 simulations have been done for $100 \mathrm{keV}$ due to high computation time.

During the step $2^{\circ}$ ) of the KMC (see section III.A), the vacancies and interstitials recombined each other before the algorithm makes them moving. The quantity of recombination experienced during this step is called in this work the initial recombination rate, defined as:

$$
\tau_{\text {init. rec. }}=\frac{\text { Nb.of rec. Frenkel pairs in } \left.2^{\circ}\right)}{\text { Total nb.of Frenkel pairs at } t=0}
$$

The mean initial recombination rate of Frenkel pairs equals to approximately $57 \%$ (see Table 2), in accordance with the MD simulations of Jay et al. [10] which found a value around $50 \%$. As the PKA energy increases, the standard deviation (which represents the data statistical spread) on the mean annealing rate and the mean initial recombination rate of Frenkel pairs decreases. This is due to the higher number of defects in the simulation volume, which increase the number of samples on which the statistical study is made. As a consequence, the statistical study tends more easily to the mean value of initial recombination and annealing rates. The results seem to be quite equivalent for $10 \mathrm{keV}$ and $100 \mathrm{keV}$ PKA. These results show that the discretization of the PKA energy according to the proton energy induces a small error on the KMC results since the trap population and the annealing rates does not vary on a large amplitude.

With regard to the average percentage of defects for each species, it is found that KMC simulations for $1 \mathrm{keV}$ PKA provide highly variable estimates. The standard deviations are large and give a significant uncertainty on the average number of defects. These values reflect the fact that at the end of a KMC simulation for example, a tri-vacancy may be present in small numbers, either majority or completely absent. For PKA of $1 \mathrm{keV}$, the tri-interstitial population is clearly a minority compared to the others. This deficit is surely explained by the fact that the interstitials have a frequency of jumps weaker than the vacancies. Therefore, highly mobile vacancies will either migrate and recombine with interstitials, or the interstitials will react with vacancy complexes that formed before interstitials started moving. Finally, like the vacancies, some interstitials can migrate away from the initial damage cascade and exit the simulation volume. Once all these interstitials are consumed, there are not enough defects to form complexes such as triinterstitials. On our own observations, the absolute fraction of defects varies from $5 \%$ to $20 \%$ in the worst case, depending on the PKA energy. We observed that a large number of defects (in terms of absolute fraction) can leave the simulation box when the PKA energy is low (around 1 $\mathrm{keV}$ ). This is due to the fact that a $1 \mathrm{keV}$ PKA damage cascade has ore chance to produce isolated defects, and consequently the recombination or association processes are more complex to achieve. The higher standard deviation on statistical study for $1 \mathrm{keV}$ PKAs is directly linked to this phenomenon.

\begin{tabular}{|c|c|c|c|}
\hline$X_{\text {rel. }}(\%)(\sigma)$ & $1 \mathrm{keV}$ & $10 \mathrm{keV}$ & $100 \mathrm{keV}$ \\
\hline$V_{2}$ & $62 \%(42 \%)$ & $50 \%(19 \%)$ & $48.6 \%(6.4 \%)$ \\
\hline$V_{3}$ & $23.5 \%(26 \%)$ & $17 \%(15.6 \%)$ & $29.8 \%(5.6 \%)$ \\
\hline$I_{3}$ & $0.75 \%(5 \%)$ & $17 \%(10.2 \%)$ & $21.6 \%(4.2 \%)$ \\
\hline
\end{tabular}

Table 3: Relative mean proportion of defects after 1 month KMC simulations for different PKA energies. The standard error is mentioned into brackets. 30 simulations have been done for $1 \mathrm{keV}$ and $10 \mathrm{keV}$, but only 5 simulations have been done for $100 \mathrm{keV}$ due to high computation time.

This study shows that the di-vacancy is the dominant defect for any PKA energy, as observed in other works [1], [4], [28], [37], [38]. For $10 \mathrm{keV}$ and $100 \mathrm{keV}$ PKA, the triinterstitials and tri-vacancies seem to be in equivalent proportion at the end of the simulation. In their work, Jay et al. identified the tri-interstitial as a main source of RTS pixels, and the tri-vacancy and larger defects (quadriinterstitial and quadri-vacancy defects) as minor RTS sources. In the literature, clusters of defects such as trivacancies or tri-interstitials are also widespread in silicon components after irradiation, and are suspected to also play a role in the degradation of these components [1], [6], [11], [39]-[41]. However, there are no measurements or calculations that clearly indicate in which proportions these 
defects are present in the irradiated silicon based components.

The average population estimated by the KMC simulations seems to be consistent with regard to the literature. The data extracted from the analysis of KMC simulations are then used to compute the Dark Current Nonuniformity.

\section{B. Methodology of the DCNU computation}

In this section, the last modeling step of our simulation chain, dedicated to the computation of the dark current increase after irradiation, is described. The original simulation Monte Carlo based toolkit described in [17], [42], [43] is used. Since it has been presented in previous works, it will not be detailed in this paper. According to incident particle energy, fluence and pixel geometry, the simulation toolkit is able to compute the mean number of nuclear interactions that occurred in pixels. From this information, a Monte Carlo simulation is made on the whole pixel array to compute the DCNU. For each nuclear interaction that occurred in a pixel, a given number of atomic displacements computed from a GEANT4 application is added to the total degradation of the pixel. This number of atomic displacements corresponds to the degradation at the initial state of the damage cascade. From the statistical study on annealing process of PKA damage structures through KMC simulations, the dark current produced by a nuclear interaction after long term annealing can be estimated.

\section{Definition of the dark current computation parameters}

According to the statistical study presented in section IV.A, the annealing rate and the typical population created following the annealing mechanism are very similar. In order to simplify the calculation, the following hypothesis is made: if the proton has energy below $10 \mathrm{MeV}$, only PKA of $1 \mathrm{keV}$ are considered. In the opposite case, if the proton has an energy greater than $10 \mathrm{MeV}$, only PKA of $10 \mathrm{keV}$ are taken into account.

At first, we consider that the annealing process that follows a nuclear reaction is not the same at each nuclear interaction that occurred in a pixel. Hence, an annealing factor is randomly drawn in a Gaussian distribution, centered on the mean annealing rate provided by the statistical study made in the previous section (see Table 2). The standard deviation of the Gaussian distribution corresponds also to the standard deviation on the KMC annealing rate data. As the data provided in Table 3 indicates relative mean proportion of defects, we propose here to convert these data in absolute values, necessary to use them as input parameters (Table 4).

\begin{tabular}{|c|c|c|c|}
\hline \multirow{2}{*}{$\boldsymbol{P K A}$} & \multicolumn{3}{|c|}{$\boldsymbol{X}(\%)$} \\
\cline { 2 - 4 } Energy & $V_{2}^{-/ 0}$ & $V_{3}^{-/ 0}$ & $I_{3}$ \\
\hline $1 \mathrm{keV}$ & 70 & 30 & 0 \\
\hline $10 \mathrm{keV}$ & 54 & 23 & 23 \\
\hline
\end{tabular}

Table 4: Average population of complex defects used to compute the dark current for different PKA energy.

Then, the electron-hole pair thermal generation rate of average population of defects estimated by the KMC algorithm is used to convert the number of atomic displacements produced by a nuclear reaction into an electrical current (equation (9)). It means that on average, one nuclear interaction produces the average population predicted by the KMC algorithm. The defect electrical parameters capture shown in Table 5 are taken as most representative from calculations and measurements available in the literature [4], [26]-[28], [30], [31], [40], [44]-[48]. More references can be found in [49]. However, just as migration parameters, electrical parameters data suffer from experimental uncertainties and their calculation depends on simulation hypothesis. For example, the capture cross sections data spread on several orders of magnitude $\left(10^{-18} \mathrm{~m}^{2}\right.$ to $\left.10^{-21} \mathrm{~m}^{2}\right)$.

\begin{tabular}{|c|c|c|c|c|}
\hline Species & $\boldsymbol{E}_{\boldsymbol{t}}(\boldsymbol{e} \boldsymbol{V})$ & $\boldsymbol{\sigma}_{\boldsymbol{n}}\left(\boldsymbol{m}^{2}\right)$ & $\boldsymbol{\sigma}_{\boldsymbol{p}}\left(\boldsymbol{m}^{2}\right)$ & $\boldsymbol{g}_{\boldsymbol{i}}(\# / \boldsymbol{s})$ \\
\hline$V_{2}^{-/ 0}$ & $E_{v}+0.71$ & $5 \times 10^{-19}$ & $5 \times 10^{-19}$ & 0.55 \\
\hline$V_{3}^{-/ 0}$ & $E_{v}+0.665$ & $2.4 \times 10^{-19}$ & $1 \times 10^{-18}$ & 6.49 \\
\hline$I_{3}$ & $E_{v}+0.92$ & $1 \times 10^{-18}$ & $1 \times 10^{-18}$ & $2.8 \times 10^{-4}$ \\
\hline
\end{tabular}

Table 5: Electrical properties of the standard complex defects used to compute the dark current for different PKA energy.

The term $g_{i}$ corresponds to the intrinsic thermal generation rate for the defect $i$, expressed in number of electron-hole pairs thermally generated by a defect $i$, and it is expressed as followed:

$$
g_{i}=\frac{\sigma_{n} \sigma_{p} v_{t h_{n}} v_{t h_{p}} n_{i}}{\sigma_{n} v_{t h_{n}} \exp \left(\frac{E_{t}-E_{F_{i}}}{k_{B} T}\right)+\sigma_{p} v_{t h_{p}} \exp \left(-\frac{E_{t}-E_{F_{i}}}{k_{B} T}\right)}
$$

where $\sigma_{n, p}$ is the electron and hole capture cross section respectively, $v_{t h_{n, p}}$ the thermal velocity of electron and hole respectively $(m / s), E_{t}$ the defect's energy level in the silicon bandgap and $E_{F_{i}}$ the intrinsic Fermi level $(\mathrm{eV})$, and $n_{i}$ the intrinsic carrier concentration $\left(\mathrm{m}^{-3}\right)$.

\section{Mathematical formalism of the DCNU computation}

In addition, a 3D Electric Field Enhancement (3D EFE) effects model is used to simulate the influence of the electric field on the electron-hole pair thermal generation rate [50]. For a nuclear reaction $j$, the dark current associated is computed as follows:

$$
\Delta I_{j}=N_{d, j} \times\left\langle g^{\prime}\right\rangle_{j} \times\left(1-A F_{j}\right)
$$

With $N_{d, j}$ the number of atomic displacements contained in the damage cascade, $A F_{j}$ the random annealing factor drawn in a Gaussian distribution, and $\left\langle g^{\prime}\right\rangle_{j}$ the thermal generation rate for the typical defect population estimated by the KMC enhanced by the 3D EFE model. The mean thermal generation rate is expressed:

$$
\left\langle g^{\prime}\right\rangle_{j}=\frac{\sum_{i=0}^{N_{\text {def. }} \cdot} \lambda_{g, j, i} \times g_{i}}{N_{\text {def. }}}
$$

Where $N_{\text {def. }}$ is the total number of defects that composed the standard population predicted by the KMC method, and $\lambda_{g, j, i}$ the electric field enhancement factor computed for each defect $i$. For each nuclear interaction an electric field is randomly drawn in the electric field distribution associated to the simulated pixel image sensor, and the electric field effects are computed for each defect. The electric field distribution comes from an electric field depth profile provided by TCAD software simulation. The term $\left(1-A F_{j}\right)$ corresponds to the fraction of initial Frenkel pairs that survives to the annealing process and produces electrically 
active stable defects. Hence, this computation gives the mean number of electron-hole pair thermally generated by unit of time (under the influence of electric field) from a fraction of primary defects that have survived and formed electrically active and stable complex defects.

\section{E. Comparison with experimental data}

According to Fig. 4, the comparison between simulations using the KMC method and those using the universal damage factor shows that, for the simulation parameters considered, results are quite equivalent. The method used to take into account the 3D EFE model with the UDF is the same as the reference [50].

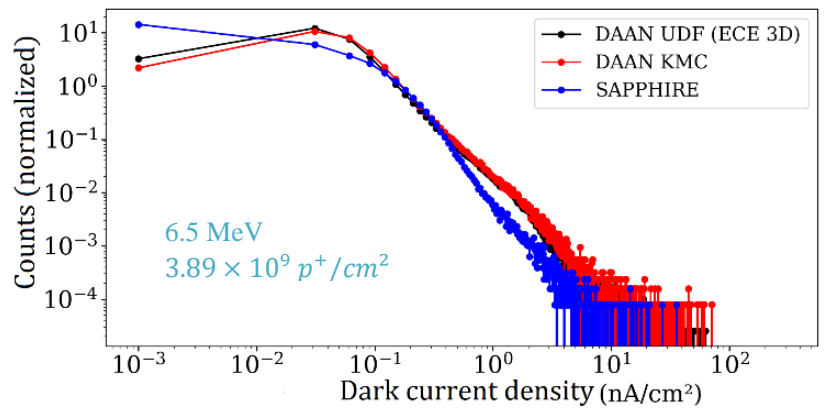

(a)

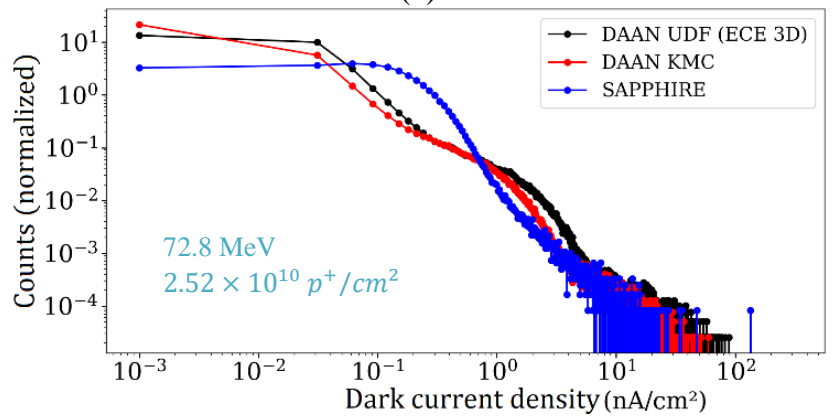

(b)

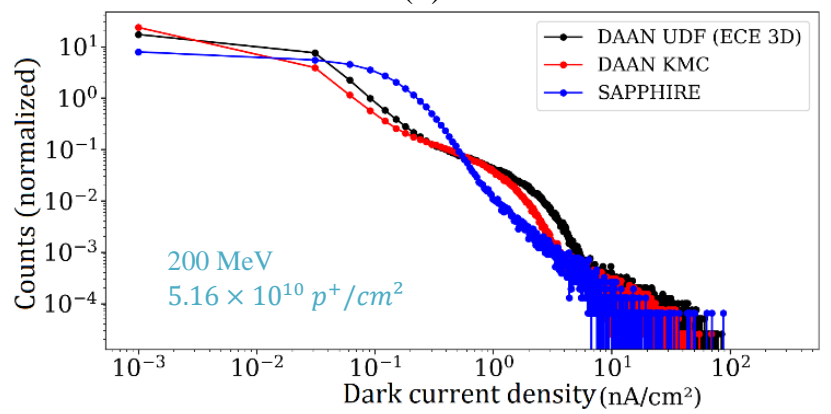

(c)

Fig. 4: Comparison between the Dark Current Nonuniformity measured experimentally (blue curve), simulated with the KMC input data (red curve) and simulated with the Universal Damage Factor for protons of (a) $6.5 \mathrm{MeV}$ at a fluence of $3.89 \times 10^{9} \mathrm{p}^{+} / \mathrm{cm}^{2}$, (b) $72.8 \mathrm{MeV}$ at a fluence of $2.52 \times 10^{10} \mathrm{p}^{+} / \mathrm{cm}^{2}$, (c) $200 \mathrm{MeV}$ at a fluence of $5.16 \times$ $10^{10} \mathrm{p}^{+} / \mathrm{cm}^{2}$. All measurements are made 1 month after irradiation at 294.15 K. The simulated image sensor contains 1310720 pixels.

For proton modeling of $6.5 \mathrm{MeV}$ (trap population and annealing factor corresponding to $1 \mathrm{keV}$ PKA), the simulation from the KMC method provided a very slight overestimation of the maximum dark current (about a factor 1.1) compared to the universal damage factor method. With regard to the estimated increase in dark current for protons of $72.8 \mathrm{MeV}$ and $200 \mathrm{MeV}$ (trap population and annealing factor corresponding to $10 \mathrm{keV}$ PKA), the dark current distributions from the KMC method show a reduction of the number of pixels and their dark current intensity located at the tail of the distribution (greater than $3 \mathrm{nA} / \mathrm{cm}^{2}$ ) compared to the UDF method. For $72.8 \mathrm{MeV}$ and $200 \mathrm{MeV}$ irradiations, the simulation of dark currents by the KMC method does not allow to reproduce the last channel of the experimental distribution, containing the few highly degraded pixels. Apart from this single point, the tail of distribution (currents greater than about $2-3 \mathrm{nA} / \mathrm{cm}^{2}$ ) is well predicted by the DAAN KMC method.

Depending on the parameters considered, the new model makes it possible to estimate the number and intensity of hot pixels in a consistent way with the experimental distribution compared to the UDF method for currents greater than $0.9 \mathrm{nA} / \mathrm{cm}^{2}$.

Another interesting results is the mean concentration of defects that can be deduced from this chain of simulation. On average, the number of Frenkel pairs for a given PKA energy $\left\langle N_{\text {Frenkel }}\right\rangle$ according to GEANT4 nuclear reaction simulations is given in the table below (100 PKA are considered to compute the average):

\begin{tabular}{|c|c|c|c|}
\cline { 2 - 4 } \multicolumn{1}{c|}{} & \multicolumn{3}{c|}{ PKA energy } \\
\cline { 2 - 4 } \multicolumn{1}{c|}{} & $1 \mathrm{keV}$ & $10 \mathrm{keV}$ & $100 \mathrm{keV}$ \\
\hline$\left\langle N_{\text {Frenkel }}\right\rangle$ & 22.1 & 167.3 & 919.2 \\
\hline
\end{tabular}

Table 6: Mean number of Frenkel pairs according to PKA energy in silicon.

The mean concentration of defects $[X]$ produced for 1 PKA in the depleted volume of the pixel $V_{\text {depleted }}$ is obtained according to the following expression:

$$
[X]=\frac{\left\langle N_{\text {Frenkel }}\right\rangle \times(1-A F) \times X}{V_{\text {depleted }}}
$$

Where $X$ is the average fraction of defects issued from Table 4. For the di-vacancy level $(X=0.7$ for a $1 \mathrm{keV}$ PKA, $X=0.54$ for a $10 \mathrm{keV} \mathrm{PKA}$ ), the mean concentration is the studied pixel is equal to $8.08 \times 10^{10} \mathrm{~cm}^{-3}$ for 1 PKA of $1 \mathrm{keV}, 3.37 \times 10^{11} \mathrm{~cm}^{-3}$ for a $10 \mathrm{keV}$ PKA, both one month after irradiation at $294.15 \mathrm{~K}$. These values obtained by KMC simulations are in the order of magnitude of experimental values of di-vacancy concentrations found in [51], [52] obtained respectively with Current Transient Spectroscopy method (CTS) and Deep Level Transient Spectroscopy (DLTS) method, at a similar Displacement Damage Dose level. Because the tri-interstitials and trivacancies have found interest recently, concentration data are rare for these defects and as a consequence, no other comparison between simulation and experimental concentrations is proposed in this work. These final results are really encouraging for the development of the simulation chain described in this work.

\section{DISCUSSION}

For this study, the hole capture cross section used in section IV.E (Table 5) for $1 \mathrm{keV}$ PKA is divided by a factor 2 for each defect to decrease the rate of generation of electron-hole pairs. The hole capture cross section has been reduced as a simple case study in order to illustrate the parametric sensitivity of the methodology proposed in this work. However, the selected values remain within the range of effective sections encountered in the literature (see references in section IV.C). The choice of hole capture cross 
section (instead of the electron one) is supported by the fact the thermal generation rate is more sensitive to the variation of hole capture cross section for an acceptor trap level when electric field enhancement effects are considered, as explained in [50].

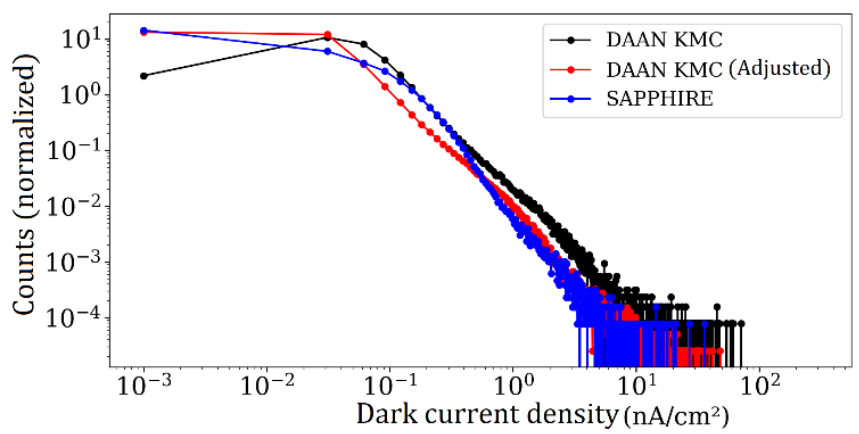

Fig. 5: Comparison between the Dark Current Nonuniformity measured experimentally (blue curve), simulated with the KMC input data with adjusted electrical parameters (red curve) and simulated with the Universal Damage Factor for protons of $6.5 \mathrm{MeV}$ at a fluence of $3.89 \times 10^{9} \mathrm{p}^{+} / \mathrm{cm}^{2}$.

Considering these adjusted parameters leads to a better estimation of the whole dark current distribution, as shown on Fig. 5. An attempt to improve the DCNU modelling has been performed by adjusting the value of the hole capture cross section. But this is not the sole parameter that could be adapted to best fit experimental data. Indeed the amplitude of the dark current depends also on other parameters such as the internal electric field distribution which remains unknown in most of tested devices. The differences between the experimental dataset and the UDF estimation could be caused by some modeling inaccuracies related for instance to the electric field distribution in the pixel, for which manufacturer information is not always readily available. The adjustment of the defects characteristics can bring the simulation closer to the experimental data but as the population of defects predicted by the KMC algorithm is not absolutely the exact one as the level of complexity is limited to tri-vacancy and tri-interstitial the relevance of their characteristics must be regarded in the scope of the hypothesis of our calculation.

In order to analyze the similarities between the KMC method and the UDF method, the average conversion factor for the KMC method and for the UDF method are evaluated. This average conversion coefficient $\alpha$ (expressed in $\mathrm{nA} / \mathrm{cm}^{2}$ for 1 displaced atom). This average coefficient is calculated by taking the total number of nuclear interactions $N_{\text {int. }}$ that occurred in the pixel matrix. The conversion factor of the KMC method in the simplified case where the electric field is zero (the term $\lambda_{g, j, i}$ of equation (8) is therefore equal to 1) is also computed. For this study, the adjusted trap parameters (previous paragraph) corresponding to a proton irradiation of $6.5 \mathrm{MeV}$ (Fig. 5) and the annealing rate associated with $1 \mathrm{keV}$ PKA are used. The average conversion factor for each method is presented in Table 7 (expressed in $\mathrm{nA} / \mathrm{cm}^{2}$ by atomic displacement):

\begin{tabular}{|c|c|c|}
\hline$\alpha(K M C)$ & $\alpha(U D F)$ & $\alpha(K M C, \varnothing E F E)$ \\
\hline \multicolumn{2}{|c|}{ (expressed in $\mathrm{nA} / \mathrm{cm}^{2}$ for 1 atomic displacement) } \\
\hline $8.22 \times 10^{-4}$ & $1.35 \times 10^{-3}$ & $3.74 \times 10^{-4}$ \\
\hline
\end{tabular}

Table 7: Mean conversion factor for different dark current computation method: the KMC method, the Universal Damage Factor method and the KMC method without three dimensional electric field effects.
On average, the conversion factor of the UDF method is 1.66 times greater than that of the KMC method. For comparison purposes, the conversion factor of the KMC method is on average 2.2 times higher for the parameter sets considered than for the KMC method without electric field effects. Knowing that the KMC and UDF methods give similar results, one may wonder if the universal damage factor could contain a small contribution of electric field effects. One have to mention that another set of defect electrical parameters (i.e. energy level and electron-hole capture cross section) could change the value of the conversion factor for each method. Nevertheless, using trap parameters representative of the literature and a methodology validated by comparison with other research work, the KMC method developed in this work is a potential alternative to the UDF method.

The fact that the simulation results provided by our KMC method are not entirely consistent with the experimental results certainly comes from the calculation method. As a first approximation, we considered that each nuclear interaction produced on average a typical population of defects represented with fixed proportions. However, we know from the statistical study carried out on the results of the Kinetic Monte Carlo algorithm that the proportion of final defects varies according to the simulations. This variation, represented by a standard deviation, is not taken into account in our calculation method. A calculation method using this standard deviation could provide a finer analysis. Finally, we considered that for high proton energies (greater than $100 \mathrm{MeV}$ ), the energy of a PKA was about $10 \mathrm{keV}$. However, this choice may be open to discussion, as higher PKA energy may be more representative of high-energy protons and present also a PKA energy spectrum. As a result, the associated population of defects and the annealing factor used may not quite correspond to the degradation undergone by the component to these energies. It should be remembered, however, that the use of PKA of $10 \mathrm{keV}$ is constrained by the computation time that prevents us from going higher in energy for PKA. In a similar way, the influence of the box boundaries conditions on the KMC simulation results will be studied in a further work. Indeed, in this work, the defects are killed when they moved out the box boundaries for computation cost reasons.

This work seems to show that the final level of defects following one month of annealing is quite independent on the initial spatial distribution of Frenkel pairs. The clustering effects seems not to be a predominant one in the damaging process. But our modelling lays on many assumptions that could hide this mechanism that could arise for high energy protons. Multiscale simulations chains, combining DFT (Density Functional Theory), Molecular dynamic and Kinetic Monte Carlo modelling, as proposed by Jay et al. [11] could better answer to this complex question.

Finally, the case of dopants must be mentioned. As a fact, the dopants could play a role in the proton-induced dark current simulations. This case is interesting, as it raises some specific problematic. Indeed, the concentration of dopants is generally lower than the number of Frenkel pairs in the simulation box. As a consequence, the presence of an atom dopant is probabilistic. To take into account the dopants properly, one have to add a probability distribution in the KMC algorithm, which has not been determined yet. This specific case will be treated in further works. This 
problematic has also been raised recently by [11]. Another interesting perspective of this work is the role of "cluster" defects on the results of KMC simulations and by extension of the dark current simulation, as also mentioned by [11].

\section{CONCLUSIONS}

A complete simulation toolkit dedicated to the computation of the Dark Current Nonuniformity in irradiated pixel arrays is proposed. Its originality consists in the use of a Kinetic Monte Carlo algorithm to estimates the annealing process that occurs after irradiation. More precisely, the KMC algorithm is used to estimates the distribution of annealing factors for Primary Knock-on Atom of different energies, and the typical complex defect population associated to these annealing processes. From the KMC simulations, the di-vacancy is clearly identified as the most representative defect after long term annealing (one month after irradiation in this study). The standard complex defect populations and the distributions of annealing factor provide by $\mathrm{KMC}$ simulations are used as input data for a numerical tool dedicated to the prediction of the Dark Current Nonuniformity. The dark current simulation results are similar to the previous state of the art, which uses the Universal Damage Factor (UDF). This is due to the fact that the conversion factor between the number of atomic displacements and the dark current are quite equivalent on average. As a consequence, the dark current computation method presented in this work could be a potential alternative to the UDF method.

\section{REFERENCES}

[1] J. R. Srour and J. W. Palko, 'Displacement Damage Effects in Irradiated Semiconductor Devices', IEEE Trans. Nucl. Sci., vol. 60, no. 3, pp. 1740-1766, Jun. 2013, doi: 10.1109/TNS.2013.2261316.

[2] J. R. Srour, C. J. Marshall, and P. W. Marshall, 'Review of displacement damage effects in silicon devices', IEEE Trans. Nucl. Sci., vol. 50, no. 3, pp. 653-670, Jun. 2003, doi: 10.1109/TNS.2003.813197.

[3] P. M. Fahey, P. B. Griffin, and J. D. Plummer, 'Point defects and dopant diffusion in silicon', Rev. Mod. Phys., vol. 61, no. 2, pp. 289-384, Apr. 1989, doi: 10.1103/RevModPhys.61.289.

[4] G. D. Watkins, 'Intrinsic defects in silicon', Mater. Sci. Semicond. Process., vol. 3, no. 4, pp. 227-235, Aug. 2000, doi: 10.1016/S1369-8001(00)00037-8.

[5] P. W. Marshall, C. J. Dale, and E. A. Burke, 'Protoninduced displacement damage distributions and extremes in silicon microvolumes charge injection device', IEEE Trans. Nucl. Sci., vol. 37, no. 6, pp. 1776-1783, Dec. 1990, doi: 10.1109/23.101191.

[6] J. R. Srour and D. H. Lo, 'Universal damage factor for radiation-induced dark current in silicon devices', IEEE Trans. Nucl. Sci., vol. 47, no. 6, pp. 2451-2459, 2000.

[7] 'Overview $\quad$ geant4.web.cern.ch'. https://geant4.web.cern.ch/ (accessed Dec. 05, 2018).

[8] M. Andersen, C. Panosetti, and K. Reuter, 'A Practical Guide to Surface Kinetic Monte Carlo Simulations', Front. Chem., vol. 7, Art. No. 3389, 2019, doi: 10.3389/fchem.2019.00202.
[9] M. Raine et al., 'Simulation of Single Particle Displacement Damage in Silicon; Part I: Global Approach and Primary Interaction Simulation', IEEE Trans. Nucl. Sci., vol. 64, no. 1, pp. 133-140, Jan. 2017, doi: 10.1109/TNS.2016.2615133.

[10] A. Jay et al., 'Simulation of Single Particle Displacement Damage in Silicon; Part II: Generation and Long-Time Relaxation of Damage Structure', IEEE Trans. Nucl. Sci., vol. 64, no. 1, pp. 141-148, Jan. 2017, doi: 10.1109/TNS.2016.2628089.

[11] A. Jay et al., 'Simulation of Single-Particle Displacement Damage in Silicon; Part III: First Principle Characterization of Defect Properties', IEEE Trans. Nucl. Sci., vol. 65, no. 2, pp. 724-731, Feb. 2018, doi: 10.1109/TNS.2018.2790843.

[12] S. Wood et al., 'Simulation of Radiation Damage in Solids', IEEE Trans. Nucl. Sci., vol. 28, no. 6, pp. 4107-4112, Dec. 1981, doi: 10.1109/TNS.1981.4335684.

[13] C. Inguimbert, S. Duzellier, T. Nuns, and F. Bezerra, 'Using Subthreshold Heavy Ion Upset Cross Section to Calculate Proton Sensitivity', IEEE Trans. Nucl. Sci., vol. 54, no. 6, pp. 2394-2399, Dec. 2007, doi: 10.1109/TNS.2007.909983.

[14] M. C. Ursule, C. Inguimbert, and T. Nuns, 'Impact of the Border Crossing Effects on the DCNU for Pixel Arrays Irradiated With High Energy Protons', IEEE Trans. Nucl. Sci., vol. 63, no. 4, pp. 2159-2167, Aug. 2016, doi: 10.1109/TNS.2016.2550619.

[15] 'Sentaurus https://www.synopsys.com/silicon/tcad/devicesimulation/sentaurus-device.html (accessed Jul. 04, 2019).

[16] M. Trochet, L. K. Béland, J.-F. Joly, P. Brommer, and N. Mousseau, 'Diffusion of point defects in crystalline silicon using the kinetic activationrelaxation technique method', Phys. Rev. B, vol. 91, no. 22, p. 224106, Jun. 2015, doi: 10.1103/PhysRevB.91.224106.

[17] C. Inguimbert, T. Nuns, E. Martin, D. Falguère, and O. Gilard, 'Monte Carlo based denu modeling', presented at the 2012 13th European Conference on Radiation and Its Effects on Components and Systems (RADECS), Biarritz, France, Sep. 2012.

[18] K. Nordlund, 'Kinetic Monte Carlo'. 2006, Accessed: Aug. 23, 2018. [Online]. Available: http://beam.acclab.helsinki.fi/ knordlun/mc/mc8nc.pd f.

[19] K. Nordlund et al., 'Primary radiation damage: A review of current understanding and models', J. Nucl. Mater., vol. 512, pp. 450-479, Dec. 2018, doi: 10.1016/j.jnucmat.2018.10.027.

[20] J. P. Balbuena, M. J. Caturla, and E. Martinez, 'Kinetic Monte Carlo Algorithms for Nuclear Materials Applications', in Handbook of Materials Modeling: Applications: Current and Emerging Materials, W. Andreoni and S. Yip, Eds. Cham: Springer International Publishing, 2018, pp. 1-22.

[21] K. Nordlund, 'Historical review of computer simulation of radiation effects in materials', J. Nucl. Mater., vol. 520, pp. 273-295, Jul. 2019, doi: 10.1016/j.jnucmat.2019.04.028.

[22] G. Garcia and J. F. L. Corre, 'A New Collision Detection Algorithm Using Octree Models', in 
Proceedings. IEEE/RSJ International Workshop on Intelligent Robots and Systems '. (IROS '89) 'The Autonomous Mobile Robots and Its Applications, Sep. 1989, pp. 93-98, doi: 10.1109/IROS.1989.637892.

[23] O.-A.-T. Edward, J. Edward, and S. Ii, Fast Collision Detection with an $\mathrm{N}$-Objects Octree, Technical Report, OSU-ACCAD-12/93-TR7, J. Edward Swan II, Aug. 1994.

[24] F. El-Mellouhi, N. Mousseau, and P. Ordejón, 'Sampling the diffusion paths of a neutral vacancy in silicon with quantum mechanical calculations', Phys. Rev. B, vol. 70 , no. 20, p. 205202, Nov. 2004, doi: 10.1103/PhysRevB.70.205202.

[25] M. M. Sokoloski, 'Structure and kinetics of defects in silicon', NASA, Washington D.C, Technical Note NASA TN D-4154, Nov. 1967. Accessed: Feb. 07, 2020. [Online]. Available: https://ntrs.nasa.gov/archive/nasa/casi.ntrs.nasa.gov/1 9670030860.pdf.

[26] J. H. Crawford, 'Radiation Effects in Diamond Lattice Semiconductors', IEEE Trans. Nucl. Sci., vol. 10, no. 5, pp. 1-10, Nov. 1963, doi: 10.1109/TNS.1963.4323298.

[27] J. C. Bourgoin and N. de Angelis, 'Radiation-induced defects in solar cell materials', Sol. Energy Mater. Sol. Cells, vol. 66, no. 1, pp. 467-477, Feb. 2001, doi: 10.1016/S0927-0248(00)00208-7.

[28] A. O. Evwaraye and E. Sun, 'Electron-irradiationinduced divacancy in lightly doped silicon', J. Appl. Phys., vol. 47, no. 9, pp. 3776-3780, Sep. 1976, doi: $10.1063 / 1.323260$

[29] M. Tang, L. Colombo, J. Zhu, and T. Diaz de la Rubia, 'Intrinsic point defects in crystalline silicon: Tight-binding molecular dynamics studiesof selfdiffusion, interstitial-vacancy recombination, and formation volumes', Phys. Rev. B, vol. 55, no. 21, pp. 14279-14289, Jun. 1997, doi: 10.1103/PhysRevB.55.14279.

[30] A. Hallén, N. Keskitalo, F. Masszi, and V. Nágl, 'Lifetime in proton irradiated silicon', J. Appl. Phys., vol. 79, no. 8, pp. 3906-3914, Apr. 1996, doi: $10.1063 / 1.361816$.

[31] S. D. Brotherton and P. Bradley, 'Defect production and lifetime control in electron and $\gamma$-irradiated silicon', J. Appl. Phys., vol. 53, no. 8, pp. 5720-5732, Aug. 1982, doi: 10.1063/1.331460.

[32] L. J. Munro and D. J. Wales, 'Defect migration in crystalline silicon', Phys. Rev. B, vol. 59, no. 6, pp. 3969-3980, Feb. 1999, doi: 10.1103/PhysRevB.59.3969.

[33] D. Maroudas and R. A. Brown, 'Calculation of thermodynamic and transport properties of intrinsic point defects in silicon', Phys. Rev. B, vol. 47, no. 23, pp. 15562-15577, Jun. 1993, doi: 10.1103/PhysRevB.47.15562.

[34] G. H. Gilmer, T. Diaz de la Rubia, D. M. Stock, and M. Jaraiz, 'Diffusion and interactions of point defects in silicon: molecular dynamics simulations', Nucl. Instrum. Methods Phys. Res. Sect. B Beam Interact. Mater. At., vol. 102, no. 1, pp. 247-255, Aug. 1995, doi: 10.1016/0168-583X(95)80150-K.

[35] Y. Kumeda, D. J. Wales, and L. J. Munro, 'Transition states and rearrangement mechanisms from hybrid eigenvector-following and density functional theory.:
Application to $\mathrm{C} 10 \mathrm{H} 10$ and defect migration in crystalline silicon', Chem. Phys. Lett., vol. 341, no. 1, pp. 185-194, Jun. 2001, doi: 10.1016/S00092614(01)00334-7.

[36] P. Pichler, 'Intrinsic Point Defects', in Intrinsic Point Defects, Impurities, and Their Diffusion in Silicon, $\mathrm{P}$. Pichler, Ed. Vienna: Springer Vienna, 2004, pp. 77227.

[37] I. D. Konozenko, A. K. Semenyuk, and V. I. Khivrich, 'Radiation Defects Created by Co60 $\gamma$-Rays in p- and n-Type Si of High Purity', Phys. Status Solidi B-Basic Solid State Phys. - PHYS STATUS SOLIDI B-BASIC SO, vol. 35, pp. 1043-1052, Jan. 1969, doi: 10.1002/pssb.19690350257.

[38] K. Gill, G. Hall, and B. MacEvoy, 'Bulk damage effects in irradiated silicon detectors due to clustered divacancies', J. Appl. Phys., vol. 82, no. 1, pp. 126136, Jul. 1997, doi: 10.1063/1.365790.

[39] C. Virmontois, 'Analyse des effets des déplacements atomiques induits par l'environnement radiatif spatial sur la conception des imageurs CMOS', Toulouse, ISAE, 2012.

[40] V. P. Markevich et al., 'Structure and electronic properties of trivacancy and trivacancy-oxygen complexes in silicon', Phys. Status Solidi A, vol. 208, no. 3, pp. 568-571, 2011, doi: 10.1002/pssa.201000265.

[41] J. W. Palko and J. R. Srour, 'Amorphous Inclusions in Irradiated Silicon and Their Effects on Material and Device Properties', IEEE Trans. Nucl. Sci., vol. 55, no. 6, pp. 2992-2999, Dec. 2008, doi: 10.1109/TNS.2008.2006751.

[42] C. Inguimbert et al., 'Modeling the Dark Current Non-Uniformity of Image Sensors With GEANT4', IEEE Trans. Nucl. Sci., vol. 61, no. 6, pp. 3323-3330, Dec. 2014, doi: 10.1109/TNS.2014.2364332.

[43] M. C. Ursule, T. Nuns, C. Inguimbert, H. Bugnet, F. Mayer, and J. Pratlong, 'Impact of the Electric Field in the Modelling of the Dark Current Nonuniformity in Pixel Arrays', in 2017 17th European Conference on Radiation and Its Effects on Components and Systems (RADECS), Geneva, Switzerland, Oct. 2017, pp. 35-41, doi: 10.1109/RADECS.2017.8696167.

[44] C. Tivarus and W. C. McColgin, 'Dark Current Spectroscopy of Irradiated CCD Image Sensors', IEEE Trans. Nucl. Sci., vol. 55, no. 3, pp. 1719-1724, Jun. 2008, doi: 10.1109/TNS.2008.919263.

[45] G. D. Watkins and J. W. Corbett, 'Defects in Irradiated Silicon: Electron Paramagnetic Resonance of the Divacancy', Phys. Rev., vol. 138, no. 2A, pp. A543-A555, Apr. 1965, doi: 10.1103/PhysRev.138.A543.

[46] Defects in Semiconductors. Academic Press, 2015.

[47] M. Petasecca, F. Moscatelli, D. Passeri, and G. U. Pignatel, 'Numerical Simulation of Radiation Damage Effects in p-Type and n-Type FZ Silicon Detectors', IEEE Trans. Nucl. Sci., vol. 53, no. 5, pp. 2971-2976, Oct. 2006, doi: 10.1109/TNS.2006.881910.

[48] R. Radu, I. Pintilie, L. C. Nistor, E. Fretwurst, G. Lindstroem, and L. F. Makarenko, 'Investigation of point and extended defects in electron irradiated silicon-Dependence on the particle energy', J. Appl. Phys., vol. 117, no. 16, p. 164503, Apr. 2015, doi: $10.1063 / 1.4918924$. 
[49] K. Lemiere, 'Réorganisation et effets des défauts en volume dans les imageurs irradiés', These en préparation, Toulouse, ISAE, 2021.

[50] K. Lemière, C. Inguimbert, and T. Nuns, 'Influence of a 3D electric field enhancement model on the Monte Carlo calculation of the dark current in pixel arrays', J. Appl. Phys., vol. 128, no. 16, p. 164502, Oct. 2020, doi: 10.1063/5.0003613.

[51] P. Hazdra, K. Brand, and J. Vobecký, 'Defect distribution in $\mathrm{MeV}$ proton irradiated silicon measured by high-voltage current transient spectroscopy', Nucl. Instrum. Methods Phys. Res. Sect. B Beam Interact. Mater. At., vol. 192, no. 3, pp. 291-300, May 2002, doi: 10.1016/S0168583X(01)01161-2.

[52] P. Arnolda, 'La création de défauts de déplacements atomiques dans le silicium et son impact sur les composants électroniques à applications spatiales', ISAE Toulouse, 2011. 